\title{
Existence Results for an Impulsive Neutral Fractional Integrodifferential Equation with Infinite Delay
}

\author{
Alka Chadha and Dwijendra N. Pandey \\ Department of Mathematics, Indian Institute of Technology Roorkee, Roorkee, Uttarakhand Pin-247667, India \\ Correspondence should be addressed to Alka Chadha; alkachaddha03@gmail.com
}

Received 24 December 2013; Revised 6 March 2014; Accepted 3 April 2014; Published 4 May 2014

Academic Editor: Kanishka Perera

Copyright (C) 2014 A. Chadha and D. N. Pandey. This is an open access article distributed under the Creative Commons Attribution License, which permits unrestricted use, distribution, and reproduction in any medium, provided the original work is properly cited.

\begin{abstract}
We consider an impulsive neutral fractional integrodifferential equation with infinite delay in an arbitrary Banach space $X$. The
\end{abstract} existence of mild solution is established by using solution operator and Hausdorff measure of noncompactness.

\section{Introduction}

In recent years, fractional calculus has becomes an active area of research due to its demonstrated applications in widespread fields of science and engineering such as mechanics, electrical engineering, medicine, biology, ecology, and many others. The memory and hereditary properties of various materials and processes can be described by a differential equation with fractional order. The fractional differential equation also describes the efficiency of nonlinear oscillations of the earthquake. The details on the theory and its applications can be found in [1-4] and references given therein.

On the other hand, many real world processes and phenomena which are subjected during their development to short-term external influences can be modeled as impulsive differential equation with fractional order which have been used efficiently in modelling many practical problems. Their duration is negligible compared with the total duration of the entire process and phenomena. Such process is investigated in various fields such as biology, physics, control theory, population dynamics, economics, chemical technology, and medicine. In addition, the improvement of the hypothesis of the functional differential equation with infinite delay relies on a choice of phase space. There are various phase spaces which have been studied. Hale and Kato in [5] introduced a common phase space $\mathscr{P}$. For more details on phase space, we refer to books by Hale and Kato [5], Hino et al. [6] and papers [7-10]. For the study of impulsive differential equation, we refer to papers $[7,8,11-18]$ and references given therein.

The purpose of this work is to establish the existence of mild solution for impulsive fractional differential equation with infinite delay:

$$
\begin{aligned}
& \mathbf{D}_{t}^{q}\left[u(t)+g\left(t, u_{t}\right)\right]=A\left[u(t)+g\left(t, u_{t}\right)\right]+\mathscr{F}_{t}^{1-q} f\left(t, u_{t}\right), \\
& t \in J=[0, T], t \neq t_{i} \\
& u_{0}=\varphi \in \mathscr{P} \text {, } \\
& \Delta u\left(t_{i}\right)=I_{i}\left(u_{t_{i}}\right), \quad i=1,2, \ldots, n, n \in \mathbb{N},
\end{aligned}
$$

where $0<T<\infty, 0<q<1, A: D(A) \subset X \rightarrow X$ is a closed and densely defined linear operator and infinitesimal generator of a solution (resolvent) operator $\left\{S_{q}(t)\right\}_{t \geq 0}$ on Banach space $X$, and $\mathbf{D}_{t}^{q}$ denotes the fractional derivative in Caputo sense and $\mathscr{F}_{t}^{1-q}$ denotes the Riemann-Liouville fractional integral operator. The history $u_{t}:(-\infty, 0] \rightarrow X$ defined by $u_{t}(s)=u(t+s)$ for $s \in(-\infty, 0]$ belongs to some abstract phase space $\mathscr{P}$ defined axiomatically and $I_{i} \in$ $C(X, X)(i=1, \ldots, n) ; 0 \leq t_{0}<t_{1}<\cdots<t_{n} \leq t_{n+1}=T$ are fixed numbers and $\Delta u(t)$ denotes the jump of the function $u$ at the point $t$, given by $\Delta u(t)=u\left(t^{+}\right)-u\left(t^{-}\right)$. The functions $f, g: J \times \mathscr{P} \rightarrow X$ are appropriate functions and satisfy some conditions to be specified later. 
In [13], authors have considered the following impulsive fractional differential equation in a Banach space of the form

$$
\begin{gathered}
\mathbf{D}_{t}^{q} u(t)=A u(t)+f(t, u(t)), \\
q \in(0,1], t \in J=[0, T], t \neq t_{i}, \\
u(0)=u_{0}, \\
u\left(t_{i}^{+}\right)=u\left(t_{i}^{-}\right)+y_{i}, \quad i=1,2, \ldots, n, n \in \mathbb{N},
\end{gathered}
$$

where $A: D(A) \subset X \rightarrow X$ is the infinitesimal generator of a $C_{0}$-semigroup $\{T(t): t \geq 0\}$ on a Banach space, $f: J \times X \rightarrow$ $X$ is continuous, and $u_{0}, y_{i}$ are the element of $X$. Authors have established some existence and uniqueness results for system (2) under the different assumptions on initial conditions.

In this work, we adopt the idea of Wang et al. [13] and establish the existence of a mild solution for the problem (1) by using the measure of noncompactness and solution operator. The tool of measure of noncompactness has been used in linear operator theory, theory of differential and integral equations, the fixed point theory, and many others. For an initial study of theory of the measure of noncompactness, we refer to book of Banaś and Goebel [19] and Akhmerov et al. [20] and papers [9, 21-25] and references given therein.

This paper is organized as follows: In Section 2 we recall some basic definitions, lemmas, and theorems. We will prove the existence of a mild solution for the system (1) in Section 3. In the last section, we shall discuss an example to illustrate the application of the abstract results.

\section{Preliminaries}

Now we provide some basic definitions, notations, theorems, lemmas, and preliminary facts which will be used throughout this paper.

Let $X$ be a Banach space and let $C([0, T] ; X)$ be the Banach space of continuous functions $u(t)$ from $[0, T]$ to $X$ equipped with the norm $\|y\|_{C}=\sup _{t \in[0, T]}\|y(t)\|_{X}$ and $L^{p}((0, T) ; X)$ denotes the Banach space of all Bochnermeasurable functions from $(0, T)$ to $X$ with the norm

$$
\|u\|_{L^{p}}=\left(\int_{(0, T)}\|u(s)\|_{X}^{p} d s\right)^{1 / p} .
$$

Assume that $0 \in \rho(A)$, that is, $A$ is invertible. Then, this permits us to define the positive fractional power $A^{\alpha}$ as closed linear operator with domain $D\left(A^{\alpha}\right) \subseteq H$ for $\alpha \in(0,1]$. Moreover, $D\left(A^{\alpha}\right)$ is dense in $H$ with the norm

$$
\|y\|_{\alpha}=\left\|A^{\alpha} y\right\|
$$

It is easy to see that $D\left(A^{\alpha}\right)$ which is dense in $X$ is a Banach space. Henceforth, we use $X_{\alpha}$ as notation of $D\left(A^{\alpha}\right)$. Also, we have that $X_{\kappa} \hookrightarrow X_{\alpha}$ for $0<\alpha<\kappa$ and, therefore, the embedding is continuous. Then, we define $X_{-\alpha}=\left(X_{\alpha}\right)^{*}$, for each $\alpha>0$. The space $X_{-\alpha}$, standing for the dual space of $X_{\alpha}$, is a Banach space with the norm $\|z\|_{-\alpha}=\left\|A^{-\alpha} z\right\|$ for $z \in X_{-\alpha}$. For more details on the fractional powers of closed linear operators, we refer to book by Pazy [26].
To consider the mild solution for the impulsive problem, we propose that the set $\mathscr{P} \mathscr{C}([0, T] ; X)=\{u:[0, T] \rightarrow X$ : $u$ is continuous at $t \neq t_{i}$ and left continuous at $t=t_{i}$ and $u\left(t_{i}^{+}\right)$exists, for all $\left.i=1, \ldots, m\right\}$. Clearly, $\mathscr{P} \mathscr{C}([0, T] ; X)$ is a Banach space endowing the norm $\|u\|_{\mathscr{P} \mathscr{C}}=\sup _{t \in[0, T]}\|u(s)\|$. For a function $u \in \mathscr{P} \mathscr{C}([0, T] ; X)$ and $i \in\{0,1, \ldots, m\}$, we define the function $\widetilde{u_{i}} \in C\left(\left[t_{i}, t_{i+1}\right], X\right)$ such that

$$
\widetilde{u_{i}}(t)= \begin{cases}u(t), & \text { for } t \in\left(t_{i}, t_{i+1}\right], \\ u\left(t_{i}^{+}\right), & \text {for } t=t_{i} .\end{cases}
$$

For $W \subset \mathscr{P} \mathscr{C}([0, T] ; X)$ and $i \in\{0,1, \ldots, m\}$, we have $\widetilde{W_{i}}=$ $\left\{\widetilde{u_{i}}: u \in W\right\}$ and following Accoli-Arzelà type criteria.

Lemma 1. A set $B \subset \mathscr{P} \mathscr{C}([0, T] ; X)$ is relatively compact in $\mathscr{P} \mathscr{C}([0, T] ; X)$ if and only if each set $\widetilde{B_{j}}(j=1,2, \ldots, m)$ is relatively compact in $C\left(\left[t_{j}, t_{j+1}\right], X\right)(j=0,1, \ldots, m)$.

For the differential equation with infinite delay, Hale and Kato [5] proposed the phase space $\mathscr{P}$ satisfying certain fundamental axioms.

Definition 2 (see [6]). A phase space $\mathscr{P}$ is a linear space which contains all the functions mapping $(-\infty, 0]$ into Banach space $X$ with a seminorm $\|\cdot\|_{\mathscr{P}}$. The fundamental axioms assumed on $\mathscr{P}$ are the following,

(A) If $u:(-\infty, a+T] \rightarrow X, T>0$ is a continuous function on $[a, a+T]$ such that $u_{a} \in \mathscr{P}$ and $\left.u\right|_{[a, a+T]} \in$ $\mathscr{P} \in \mathscr{P} \mathscr{C}([a, a+T] ; X)$, then for every $t \in[a, a+T)$, the following conditions hold:

(i) $u_{t} \in \mathscr{P}$,

(ii) $H\left\|u_{t}\right\|_{\mathscr{P}} \geq\|u(t)\|$,

(iii) $\left\|u_{t}\right\|_{\mathscr{P}} \leq N(t+a)\left\|u_{a}\right\|_{\mathscr{P}}+K(t-a) \sup \{\|u(s)\|$ : $a \leq s \leq t\}$.

Where $H$ is a positive constant, $N, K:[0, \infty) \rightarrow$ $[0, \infty), N$ is a locally bounded, $K$ is continuous, and $H, N, K$ are independent of $u(\cdot)$.

(A1) For the function $u$ in (A1), $u_{t}$ is a $\mathscr{P}$-valued continuous function for $t \in[a, a+T]$.

(B) The space $\mathscr{P}$ is complete.

Now, we state some basic definitions and properties of fractional calculus.

Mittag-Leffler. The definition of one parameter Mittag-Leffler function is given as

$$
E_{\alpha}(z)=\sum_{k=0}^{\infty} \frac{z^{k}}{\Gamma(\alpha k+1)},
$$

and two-parameter Mittag-Leffler function is defined as

$$
\begin{array}{r}
E_{\alpha, \beta}(z)=\sum_{k=0}^{\infty} \frac{z^{k}}{\Gamma(\alpha z+\beta)}=\frac{1}{2 \pi i} \int_{C} \frac{\mu^{\alpha-\beta} e^{\mu}}{\mu^{a}-z} d \mu, \\
\alpha, \beta>0, z \in \mathbb{C},
\end{array}
$$


where $C$ is a contour which starts and ends at $-\infty$ and encircles the disc $|\mu| \leq|z|^{1 / 2}$ counter clockwise. The Laplace transform of the Mittag-leffler is defined as

$$
L\left(t^{\beta-1} E_{\alpha, \beta}\left(-\rho^{\alpha} t^{\alpha}\right)\right)=\frac{\lambda^{\alpha-\beta}}{\lambda^{\alpha}+\rho^{\alpha}}, \quad \operatorname{Re} \lambda>\rho^{1 / \alpha}, \rho>0 .
$$

For more details we refer to [1].

Laplace transform of integer order derivatives is defined as

$$
\begin{aligned}
L\left[F^{n}(t) ; \lambda\right] & =\lambda^{n} L[F(t)]-\sum_{k=0}^{n-1} \lambda^{n-k-1} F^{k}(0) \\
& =\lambda^{n} L[F(t)]-\sum_{k=0}^{n-1} \lambda^{k} F^{n-k-1}(0) .
\end{aligned}
$$

Definition 3. The Riemann-Liouville fractional integral operator $\mathscr{I}$ is defined as

$$
\mathscr{F}_{t}^{q} F(t)=\frac{1}{\Gamma(q)} \int_{0}^{t}(t-s)^{q-1} F(s) d s,
$$

where $F \in L^{1}((0, T) ; X)$ and $q>0$ is the order of the fractional integration.

Definition 4. The Riemann-Liouville fractional derivative is given as

$$
\mathbf{D}_{t}^{q} F(t)=D_{t}^{n} \mathcal{F}_{t}^{n-q} F(t), \quad n-1<q<n, n \in \mathbb{N},
$$

where $D_{t}^{n}=d^{n} / d t^{n}, F \in L^{1}((0, T) ; X)$, and $\mathscr{I}_{t}^{n-q} F \in$ $W^{n, 1}((0, T) ; X)$. Here, the notation $W^{n, 1}((0, T) ; X)$ stands for the Sobolev space defined as

$$
\begin{aligned}
& W^{n, 1}((0, T) ; X) \\
& =\left\{y \in X: \exists z \in L^{1}((0, T) ; X):\right. \\
& \left.y(t)=\sum_{k=0}^{n-1} d_{k} \frac{t^{k}}{k !}+\frac{t^{n-1}}{(n-1) !} * z(t), t \in(0, T)\right\} .
\end{aligned}
$$

Note that $z(t)=y^{n}(t)$ and $d_{k}=y^{k}(0)$.

Definition 5. The Caputo fractional derivative is given as

$$
\mathbf{D}_{t}^{\alpha} F(t)=\frac{1}{\Gamma(n-\alpha)} \int_{0}^{t}(t-s)^{n-\alpha-1} F^{n}(s) d s, \quad n-1<\alpha<n,
$$

where $F \in C^{n-1}((0, T) ; X) \cap L^{1}((0, T) ; X)$ and the following holds

$$
\mathscr{F}_{t}^{q}\left(\mathbf{D}_{t}^{q} F(t)\right)=F(t)-\sum_{k=0}^{n-1} \frac{t^{k}}{k !} F^{k}(0)
$$

Definition 6 (see [27]). A family $\left\{S_{q}(t)\right\}_{t \geq 0} \subset \mathscr{L}(X)$ of bounded linear operators in $X$ is called a resolvent (or solution operator) generating by $A$ if the following conditions are fulfilled:

(1) $S_{q}(t)$ is strongly continuous on $\mathbb{R}^{+}$and $S_{q}(0)=I$;

(2) for $x \in D(A)$ and $t \geq 0, S_{q}(t) D(A) \subset D(A)$ and $A S_{q}(t) x=S_{q}(t) A x$

(3) $S_{q}(t) x$ is the solution of the equation

$$
\begin{array}{r}
u(t)=x+\frac{1}{\Gamma(q)} \int_{0}^{t}(t-s)^{q-1} A u(s) d s, \\
\forall x \in D(A), t \geq 0,
\end{array}
$$

where $\mathscr{L}(X)$ denotes the space of all bounded linear operators from $X$ into $X$ endowed with the norm of operators. [27])

Also, the solution operator $S_{q}(t)$ for (15) is defined as (see

$$
\lambda^{q-1}\left(\lambda^{q} I-A\right)^{-1} x=\int_{0}^{\infty} e^{-\lambda t} S_{q}(t) x d t, \quad \operatorname{Re} \lambda>\omega, x \in X,
$$

where $\omega \geq 0$ and $\left\{\lambda^{q}: \operatorname{Re} \lambda>\omega\right\} \subset \rho(A)$.

Let

$$
\sum(\omega, \theta)=\{\lambda \in \mathbb{C}:|\arg (\lambda-\omega)|<\theta\} .
$$

Definition 7 (see [27]). A solution operator $S_{q}(t)$ is said to be analytic if $S_{q}(\cdot): \mathbb{R}^{+} \rightarrow \mathscr{L}(X)$ admits analytic extension to a sector $\sum\left(0, \theta_{0}\right)$ for some $0<\theta_{0} \leq \pi / 2$. Furthermore, $A n$ analytic resolvent $S_{q}(t)$ is said to be of analyticity type $\left(\omega_{0}, \theta_{0}\right)$ if, for $\theta_{0}>\theta$ and $\omega_{0}<\omega$, there exists $M=M(\omega, \theta)$ such that $\left\|S_{q}(t)\right\| \leq M e^{\omega \operatorname{Re} z}$ for $z \in \sum(0, \theta)$; here $\operatorname{Re} z$ means the real part of $z$.

In this work, we assume that solution operator $\left\{S_{q}(t)\right\}_{t \geq 0}$ is analytic; that is, $\left\{S_{q}(t)\right\}_{t \geq 0}$ satisfy the following property.

(HA) The map $t \mapsto S_{q}(t)$ is continuous from $[0, T]$ to $\mathscr{L}(X)$ endowed with the uniform operator norm $\|\cdot\|_{\mathscr{L}(X)}$.

Without loss of generality, we have that there exist a positive constant $M$ such that $\left\|S_{q}(t)\right\| \leq M$, for $t \geq 0$.

Definition 8 (see [19]). The Hausdorff measure of noncompactness $\chi_{Z}$ is defined as

$$
\begin{aligned}
\chi_{Z}(F)=\inf \{\epsilon>0: & F \text { can be covered by finite } \\
& \text { number of balls with radius } \epsilon\},
\end{aligned}
$$

for bounded set $F \subset Z$, where $Z$ is a Banach space.

Lemma 9 (see [19]). For any bounded set $U, V \subset Y$, where $Y$ is a Banach space. Then, the following properties are fulfilled:

(i) $\chi_{Y}(U)=0$ if and only if $U$ is pre-compact; 
(ii) $\chi_{Y}(U)=\chi_{Y}(\operatorname{conv} \mathrm{U})=\chi_{\mathrm{Y}}(\overline{\mathrm{U}})$, where conv $\mathrm{U}$ and $\bar{U}$ denotes the convex hull and closure of $U$, respectively;

(iii) $\chi_{Y}(U) \subset \chi_{Y}(V)$, when $U \subset V$;

(iv) $\chi_{Y}(U+V) \leq \chi_{Y}(U)+\chi_{Y}(V)$, where $U+V=\{u+v$ : $u \in U, v \in V\}$

(v) $\chi_{Y}(U \cup V) \leq \max \left\{\chi_{Y}(U), \chi_{Y}(V)\right\}$;

(vi) $\chi_{Y}(\lambda U)=\lambda \cdot \chi_{Y}(U)$, for any $\lambda \in \mathbb{R}$;

(vii) if the map $P: D(P) \subset Y \rightarrow \mathscr{Z}$ is continuous and satisfies the Lipschitsz condition with constant $\kappa$, then we have that $\chi_{\mathscr{E}}(P U) \leq \kappa \chi_{Y}(U)$ for any bounded subset $U \subset D(P)$, where $Y$ and $\mathscr{Z}$ are Banach space.

The details on the measure of noncompactness and its applications can be found in a book by Banaś and Goebel [19] and papers $[9,10,21,23,24]$.

Lemma 10 (see [19]). A bounded and continuous map Q : $D \subset Z \rightarrow Z$ is a $\chi_{Z}$-contraction if there exists a constant $0<\kappa<1$ such that $\chi_{Z}(Q(U)) \leq \kappa \chi_{Z}(U)$, for any bounded closed subset $U \subset D$, where $Z$ is a Banach space.

Lemma 11 (see [28]). Let $D \subset Z$ be closed and convex with $0 \in D$ and let the continuous map $Q: D \rightarrow D$ be a $\chi_{Z^{-}}$ contraction. If the set $\{u \in D: u=\lambda Q u$, for $0<\lambda<1\}$ is bounded, then the map $Q$ has a fixed point in $D$.

Lemma 12 ((Darbo-Sadovskii) [19]). Let $D \subset Z$ be bounded, closed, and convex. If the continuous map $Q: D \rightarrow D$ is a $\chi_{Z}$-contraction, then the map $Q$ has a fixed point in $D$.

In this work, we consider that $\chi$ denotes the Hausdorff measure of noncompactness in $X, \chi_{C}$ denotes the Hausdorff measure in noncompactness of $C([0, T] ; X)$ and $\chi_{\mathscr{P} \mathscr{C}}$ denotes the Hausdorff measure of noncompactness in $\mathscr{P} \mathscr{C}([0, T] ; X)$.

Lemma 13 (see $[19,21])$. If $U$ is bounded subset of $C([0, T] ; X)$. Then, one has that $\chi(U(t)) \leq \chi_{C}(U)$, for all $t \in[0, T]$, where $U(t)=\{u(t) ; u \in U\} \subseteq X$. Furthermore, if $U$ is equicontinuous on $[0, T]$, then $\chi(U(t))$ is continuous on the interval $[0, T]$ and

$$
\chi_{C}(U)=\sup _{t \in[0, T]}\{\chi(U(t))\} .
$$

Lemma 14 (see [19]). If $U \subset C([0, T] ; X)$ is bounded and equicontinuous set, then $\chi(U(t))$ is continuous and

$$
\chi\left(\int_{0}^{t} U(s) d s\right) \leq \int_{0}^{t} \chi(U(s)) d s, \quad \forall t \in[0, T],
$$

where $\int_{0}^{t} U(s) d s=\left\{\int_{0}^{t} u(s) d s, u \in U\right\}$.

Lemma 15 (see [29]). (1) If $U \subset \mathscr{P} \mathscr{C}([0, T] ; X)$ is bounded, then $\chi(U(t)) \leq \chi_{\mathscr{P} \mathscr{C}}(U)$, for all $t \in[0, T]$, where $U(t)=$ $\{u(t): u \in U\} \subset X$.

(2) If $U$ is piecewise equicontinuous on $[0, T]$, then $\chi(U(t))$ is piecewise continuous for $t \in[0, T]$ and

$$
\chi_{\mathscr{P} \mathscr{C}}(U)=\sup \{\chi(U(t)): t \in[0, T]\} .
$$

(3) If $U \subset \mathscr{P} \mathscr{C}([0, T] ; X)$ is bounded and equicontinuous, then $\chi(U(t))$ is piecewise continuous for $t \in[0, T]$ and

$$
\chi\left(\int_{0}^{t} U(s) d s\right) \leq \int_{0}^{t} \chi(U(s)) d s, \quad \forall t \in[0, T],
$$

where $\int_{0}^{t} U(s) d s=\left\{\int_{0}^{t} u(s) d s: u \in U\right\}$.

\section{Main Results}

In this section, we will establish the existence results of solution for (1) by using solution operator and Hausdorff's measure of noncompactness.

From [13], we adopt the following concept of solution for impulsive differential problem (1).

Definition 16. A piecewise continuous function $u$ : $(-\infty, T] \rightarrow X$ is said to be a mild solution for impulsive problem (1) if $u_{0}=\varphi,\left.u(\cdot)\right|_{J} \in \mathscr{P} \mathscr{C}$ and

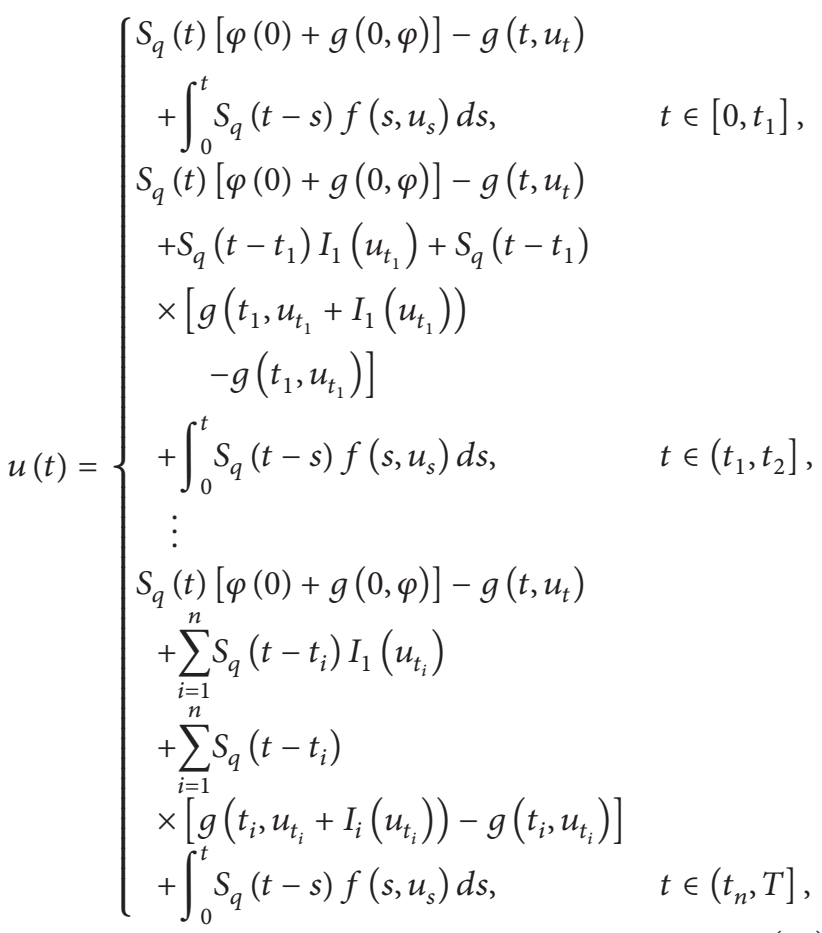

where

$$
S_{q}(t)=\frac{1}{2 \pi i} \int_{\Gamma} e^{\lambda t} \lambda^{q-1} R\left(\lambda^{q}, A\right) d \lambda
$$

Now we list the following assumptions which are required to establish main results.

(Hf) The function $f: J \times \mathscr{P} \rightarrow X$ satisfies the following conditions:

(1) the function $f(\cdot, u): J \rightarrow X$ is strongly measurable for every $u \in \mathscr{P}$ and $u_{0} \in \mathscr{P}$, $\left.u\right|_{J} \in \mathscr{P} \mathscr{C}$

(2) $f(t, \cdot): \mathscr{P} \rightarrow X$ is a continuous function for each $t \in J$; 
(3) there exists an integrable function $m_{f}$ : $[0, T] \rightarrow[0, \infty)$ and a nondecreasing continuous function $\Omega:[0, \infty) \rightarrow(0, \infty)$ such that

$$
\|f(\tau, x)\| \leq m_{f}(\tau) \Omega\left(\|x\|_{\mathscr{P}}\right), \quad \tau \in J, x \in \mathscr{P} ;
$$

(4) there exists an integrable function $\eta:[0, T] \rightarrow$ $[0, \infty)$ such that, for any bounded set $B \subset \mathscr{P}$, we have

$$
\chi\left(S_{q}(\tau) f(\tau, B)\right) \leq \eta(\tau) \sup _{-\infty \leq \theta \leq 0} \chi(B(\theta))
$$

for almost everywhere $\tau \in J$, where $B(\theta)=\{u(\theta)$ : $u \in B\}$.

(Hg) (1) For $0<\beta<1, A^{\beta} g(\cdot, \cdot)$ is Lipschitz continuous function for all $(t, v) \in J \times \mathscr{P}$ and there exist positive constants $C_{1}$ and $C_{2}$ such that

$$
\left\|A^{\beta} g(t, u)\right\| \leq C_{1}\|u\|_{\mathscr{P}}+C_{2}
$$

(2) there exists a constant $L_{g}>0$ such that

$$
\left\|A^{\beta} g(t, u)-A^{\beta} g(t, v)\right\| \leq L_{g}\|u-v\|_{\mathscr{P}},
$$

for all $u, v \in \mathscr{P}$.

(HI) The functions $I_{i}: X \quad \rightarrow \quad X,(i=1, \ldots, n)$ are continuous functions and satisfy the following conditions:

(1) There is a constant $L_{I}>0$ such that

$$
\left\|I_{i}(u)-I_{i}(v)\right\| \leq L_{I}\|u-v\|_{\mathscr{P}}, \quad \forall u, v \in \mathscr{P} .
$$

(2) There exist positive constants $L_{j}(j=1,2)$ such that

$$
\left\|I_{i}(u)\right\|=L_{1}\|u\|_{\mathscr{P}}+L_{2},
$$

for all $u \in \mathscr{P}$.

$(\mathrm{H} 1)$

$$
\frac{K_{T} M}{1-\zeta_{2}} \int_{0}^{t} m_{f}(s) d s<\int_{d}^{\infty} \frac{d s}{\Omega(s)}
$$

where

$$
\begin{gathered}
\zeta_{1}=\left(M K_{T} H+N_{T}+M C_{1}\left\|A^{-\beta}\right\| K_{T}\right)\|\varphi\|_{\mathscr{P}} \\
+K_{T} C_{2}\left\|A^{-\beta}\right\|(M+1)+K_{T}\left(n M L_{2}\left(1+L_{g}\right)\right), \\
\zeta_{2}=\left[\left\|A^{-\beta}\right\| C_{1}+n M L_{1}\left(1+L_{g}\right)\right] K_{T}<1, \quad d=\frac{\zeta_{1}}{1-\zeta_{2}} ;
\end{gathered}
$$

$(\mathrm{H} 2)$

$$
K_{T}\left[\left\|A^{-\beta}\right\| L_{g}+n M L_{I}+M n L_{g}\left(2+L_{I}\right)\right]+\int_{0}^{t} \eta(s) d s<1 .
$$

Now, let $z:(-\infty, T] \rightarrow X$ be a function given by $z_{0}=\varphi$ and $z(t)=S_{q}(t) \varphi(0)$ on $J$. It is easy to see that $\left\|z_{t}\right\|_{\mathscr{P}} \leq\left(K_{T} M H+\right.$ $\left.N_{T}\right)\|\varphi\|_{\mathscr{P}}$, where $K_{T}=\sup _{0 \leq t \leq T} K(t), N_{T}=\sup _{0 \leq t \leq T} N(t)$.

Theorem 17. Suppose that hypotheses (HA), (Hf), $(H g),(H I)$, and $(H 1)-(H 2)$ are satisfied. Then, there exists a mild solution for the impulsive problem (1).

Proof. Consider the space $S(T)=\left\{u:(-\infty, T] \rightarrow X: u_{0}=\right.$ $\left.0,\left.u\right|_{J} \in \mathscr{P}\right\}$ endowed with supremum norm $\|\cdot\|_{\mathscr{P}}$. Define the operator $Q: S(T) \rightarrow S(T)$ by

$(Q x)(t)$

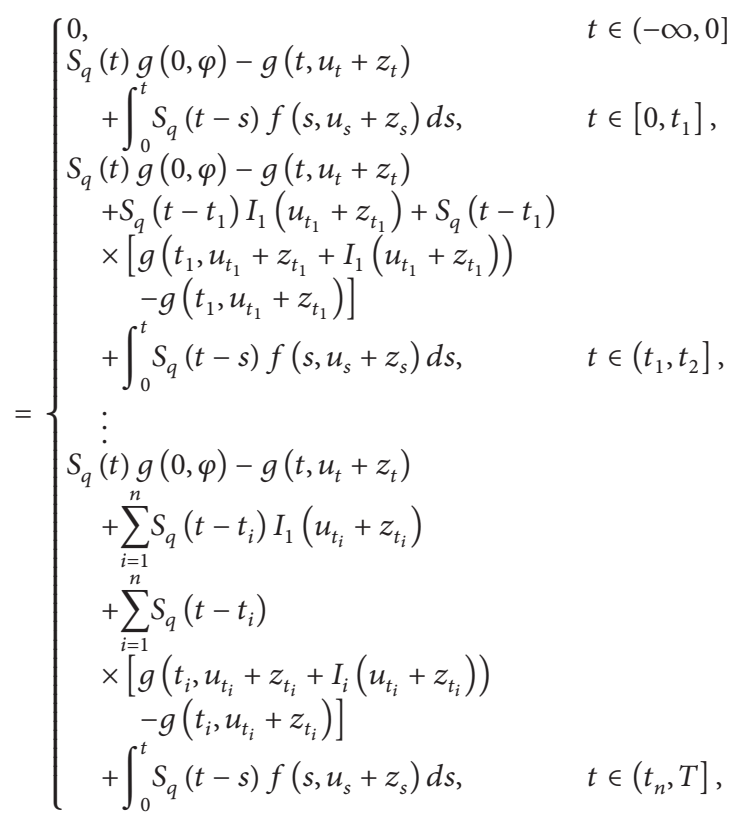

and we have that

$$
\begin{aligned}
&\left\|u_{t}+z_{t}\right\|_{\mathscr{P}} \\
& \leq\left\|u_{t}\right\|_{\mathscr{P}}+\left\|z_{t}\right\|_{\mathscr{P}}, \\
& \leq K(t) \sup \{\|u(s)\|: 0 \leq s \leq t\}+N(t)\left\|u_{0}\right\|_{\mathscr{P}} \\
&+K(t) \sup \{\|z(s)\|: 0 \leq s \leq t\}+N(t)\left\|z_{0}\right\|_{\mathscr{P}}, \\
& \leq K(t)\|u\|_{t}+K(t) M H\left\|_{\varphi}\right\|_{\mathscr{P}}+N(t)\|\varphi\|_{\mathscr{P}}, \\
& \leq\left(K_{T} M H+N_{T}\right)\|\varphi\|_{\mathscr{P}}+K_{T}\|u\|_{t},
\end{aligned}
$$

where $\|u\|_{t}=\sup _{0 \leq s \leq t}\|u(s)\|$. Thus, $Q$ is well defined and with the values in $S(T)$ by our assumptions. By Lebesgue dominated convergence theorem, axioms of phase space and assumptions (Hf), (Hg), and (HI), it is clear that $Q$ is continuous map. Furthermore, by uniformly continuity of the map $t \mapsto S_{q}(t)$ on $(0, T]$, we obtain that set $Q(S(T))$ is equicontinuous. We prove the result in following steps.

Step 1. The set $\{u \in \mathscr{P} \mathscr{C}: u=\lambda Q u$, for $0<\lambda<1\}$ is bounded.

Let $u_{\lambda}$ be a solution of $u=\lambda Q u$ for $0<\lambda<1$. Therefore, we have that

$$
\left\|u_{\lambda t}+z_{t}\right\|_{\mathscr{P}} \leq\left(K_{T} M H+N_{T}\right)\|\varphi\|_{\mathscr{P}}+K_{T}\left\|u_{\lambda}\right\|_{t} .
$$


Take $v_{\lambda}(t)=\left(K_{T} M H+N_{T}\right)\|\varphi\|_{\mathscr{P}}+K_{T}\left\|u_{\lambda}\right\|_{t}$, for $t \in J$. Then, we get that for $t \in\left[0, t_{1}\right]$

$$
\begin{aligned}
\left\|u_{\lambda}(t)\right\|= & \left\|\lambda Q u_{\lambda}(t)\right\| \leq\left\|Q u_{\lambda}(t)\right\|, \\
\leq & M\left\|A^{-\beta}\right\|\left(C_{1}\|\varphi\|_{\mathscr{P}}+C_{2}\right)+\left\|A^{-\beta}\right\|\left(C_{1} v_{\lambda}(t)+C_{2}\right) \\
& +M \int_{0}^{t} m_{f}(s) \Omega\left(v_{\lambda}(s)\right) d s .
\end{aligned}
$$

For $t \in\left(t_{1}, t_{2}\right]$, we have

$$
\begin{aligned}
\left\|u_{\lambda}(t)\right\| \leq & M\left\|A^{-\beta}\right\|\left(C_{1}\|\varphi\|_{\mathscr{P}}+C_{2}\right) \\
& +\left\|A^{-\beta}\right\|\left(C_{1} v_{\lambda}(t)+C_{2}\right)+M\left\|I_{1}\left(v_{\lambda}\left(t_{1}\right)\right)\right\| \\
& +M\left\|g\left(t_{1}, v_{\lambda}\left(t_{1}\right)+I_{1}\left(v_{\lambda}\left(t_{1}\right)\right)\right)-g\left(t_{1}, v_{\lambda}\left(t_{1}\right)\right)\right\| \\
& +M \int_{0}^{t} m_{f}(s) \Omega\left(v_{\lambda}(s)\right) d s, \\
\leq & M\left\|A^{-\beta}\right\|\left(C_{1}\|\varphi\|_{\mathscr{P}}+C_{2}\right)+\left\|A^{-\beta}\right\|\left(C_{1} v_{\lambda}(t)+C_{2}\right) \\
& +M\left(L_{1}\left(v_{\lambda}(t)\right)+L_{2}\right) \\
& +M L_{g}\left(L_{1} v_{\lambda}(t)+L_{2}\right) \\
& +M \int_{0}^{t} m_{f}(s) \Omega\left(v_{\lambda}(s)\right) d s .
\end{aligned}
$$

For $t \in\left(t_{n}, T\right]$,

$$
\begin{aligned}
\left\|u_{\lambda}(t)\right\| \leq & M\left\|A^{-\beta}\right\|\left(C_{1}\|\varphi\|_{\mathscr{P}}+C_{2}\right)+\left\|A^{-\beta}\right\|\left(C_{1} v_{\lambda}(t)+C_{2}\right) \\
& +n M\left(L_{1}\left(v_{\lambda}(t)\right)+L_{2}\right)+n M L_{g}\left(L_{1} v_{\lambda}(t)+L_{2}\right) \\
& +M \int_{0}^{t} m_{f}(s) \Omega\left(v_{\lambda}(s)\right) d s .
\end{aligned}
$$

Therefore, for all $t \in[0, T]=J$, we have

$$
\begin{aligned}
\left\|u_{\lambda}(t)\right\| \leq & M\left\|A^{-\beta}\right\|\left(C_{1}\|\varphi\|_{\mathscr{P}}+C_{2}\right) \\
& +\left\|A^{-\beta}\right\|\left(C_{1} v_{\lambda}(t)+C_{2}\right)+n M L_{1}\left(1+L_{g}\right) v_{\lambda}(t) \\
& +n M L_{2}\left(1+L_{g}\right)+M \int_{0}^{t} m_{f}(s) \Omega\left(v_{\lambda}(s)\right) d s, \\
\leq & M\left\|A^{-\beta}\right\|\left(C_{1}\|\varphi\|_{\mathscr{P}}+C_{2}\right) \\
& +\left\|A^{-\beta}\right\| C_{2}+n M L_{2}\left(1+L_{g}\right) \\
& +\left(\left\|A^{-\beta}\right\| C_{1}+n M L_{1}\left(1+L_{g}\right)\right) v_{\lambda}(t) \\
& +M \int_{0}^{t} m_{f}(s) \Omega\left(v_{\lambda}(s)\right) d s .
\end{aligned}
$$

From $v_{\lambda}(t)=\left(K_{T} M H+N_{T}\right)\|\varphi\|_{\mathscr{P}}+K_{T}\left\|u_{\lambda}\right\|_{t}$, it implies that

$$
\begin{aligned}
v_{\lambda}(t) \leq & \left(M K_{T} H+N_{T}+M\left\|A^{-\beta}\right\| C_{1} K_{T}\right)\|\varphi\|_{\mathscr{P}} \\
& +K_{T}\left\|A^{-\beta}\right\| C_{2}(M+1)+K_{T}\left(n M L_{2}\left(1+L_{g}\right)\right) \\
& +K_{T}\left(\left\|A^{-\beta}\right\| C_{1}+n M L_{1}\left(1+L_{g}\right)\right) v_{\lambda}(t) \\
& +M K_{T} \int_{0}^{t} m_{f}(s) \Omega\left(v_{\lambda}(s)\right) d s,
\end{aligned}
$$

and consequently,

$$
\begin{aligned}
v_{\lambda}(t) & \leq \frac{\zeta_{1}}{1-\zeta_{2}}+\frac{M K_{T}}{1-\zeta_{2}} \int_{0}^{t} m_{f}(s) \Omega\left(v_{\lambda}(s)\right) d s, \\
& =d+\frac{M K_{T}}{1-\zeta_{2}} \int_{0}^{t} m_{f}(s) \Omega\left(v_{\lambda}(s)\right) d s,
\end{aligned}
$$

where

$$
\begin{aligned}
\zeta_{1}= & \left(M K_{T} H+N_{T}+M C_{1}\left\|A^{-\beta}\right\| K_{T}\right)\|\varphi\|_{\mathscr{P}} \\
& +K_{T}\left\|A^{-\beta}\right\| C_{2}(M+1)+n K_{T} M L_{2}\left(1+L_{g}\right), \\
& \zeta_{2}=K_{T}\left[\left\|A^{-\beta}\right\| C_{1}+n M L_{1}\left(1+L_{g}\right)\right] .
\end{aligned}
$$

Let $\xi_{\lambda}(t)=d+\left(M K_{T} /\left(1-\zeta_{2}\right)\right) \int_{0}^{t} m_{f}(s) \Omega\left(\xi_{\lambda}(s)\right) d s$. Thus $\xi_{\lambda}(0)=d$. Therefore, we get

$$
\frac{\xi_{\lambda}^{\prime}(t)}{\Omega\left(\xi_{\lambda}(t)\right)} \leq \frac{K_{T} M}{1-\zeta_{2}} m_{f}(t) .
$$

Integrating above inequality we have that

$$
\int_{d}^{\xi_{\lambda}(t)} \frac{d s}{\Omega(s)} \leq \frac{K_{T} M}{1-\zeta_{2}} \int_{0}^{t} m_{f}(s) d s<\int_{d}^{\infty} \frac{d s}{\Omega(s)} .
$$

It gives that the functions $\xi_{\lambda}(t)$ are bounded on interval $J$. Therefore, the functions $v_{\lambda}(t)$ are bounded and $u_{\lambda}(\cdot)$ are also bounded on $J$.

Step 2. The map $Q$ is a $\chi$-contraction.

Firstly, we introduce decomposition of $Q$ into $Q=Q_{1}+$ $Q_{2}$, for $t \geq 0$ such that

$$
\begin{gathered}
Q_{1} u(t)=\left\{\begin{array}{c}
S_{q}(t) g(0, \varphi)-g\left(t, u_{t}+z_{t}\right), \\
S_{q}(t) g(0, \varphi)-g\left(t, u_{t}+z_{t}\right) \\
+\sum_{i=1}^{m} S_{q}\left(t-t_{i}\right) I_{i}\left(u_{t_{i}}+z_{t_{i}}\right)+\sum_{i=1}^{m} S_{q}\left(t-t_{i}\right) \\
\times\left[0, t_{1}\right], \\
\quad-g\left(t_{i}, u_{t_{i}}+z_{t_{i}}+I_{i}\left(u_{t_{i}}+z_{t_{i}}\right)\right)
\end{array}\right. \\
\left.\left.\quad Q_{2} u(t)=\int_{0}^{t} S_{t_{i}}+z_{t_{i}}\right)\right], \\
t \in\left(t_{m}, t_{m+1}\right], m=1,2,3, \ldots, n ;
\end{gathered}
$$


To prove the result, we firstly show that $Q_{1}$ is Lipschitz continuous. For $x_{1}, x_{2} \in S(T)$ and $t \in\left[0, t_{1}\right]$, we have that

$$
\begin{aligned}
\left\|Q_{1} x_{1}(t)-Q_{1} x_{2}(t)\right\| & =\left\|g\left(t, x_{1 t}+z_{t}\right)-g\left(t, x_{2 t}+z_{t}\right)\right\|, \\
& \leq\left\|A^{-\beta}\right\| L_{g}\left\|x_{1 t}-x_{2 t}\right\| .
\end{aligned}
$$

For $t \in\left(t_{m}, t_{m+1}\right], m=1,2, \ldots, n$ we have

$$
\begin{aligned}
\left\|Q_{1} x_{1}(t)-Q_{1} x_{2}(t)\right\| \\
\leq\left\|A^{-\beta}\right\| L_{g}\left\|x_{1 t}-x_{2 t}\right\|_{\mathscr{P}}+m M L_{I}\left\|x_{1 t}-x_{2 t}\right\|_{\mathscr{P}} \\
\quad+M m L_{g}\left(2+L_{I}\right)\left\|x_{1 t}-x_{2 t}\right\|_{\mathscr{P}} \\
=K_{T}\left[\left\|A^{-\beta}\right\| L_{g}+m M L_{I}+M m L_{g}\left(2+L_{I}\right)\right] \\
\quad \times\left\|x_{1}-x_{2}\right\|_{T} .
\end{aligned}
$$

Thus for $t \in[0, T]$ we have

$$
\begin{aligned}
& \left\|Q_{1} x_{1}(t)-Q_{1} x_{2}(t)\right\| \\
& \leq K_{T}\left[\left\|A^{-\beta}\right\| L_{g}+n M L_{I}+M n L_{g}\left(2+L_{I}\right)\right] \\
& \quad \times\left\|x_{1}-x_{2}\right\|_{T} .
\end{aligned}
$$

Taking supremum on $[0, T]$, we get

$$
\begin{aligned}
& \left\|Q_{1} x_{1}-Q_{1} x_{2}\right\|_{T} \\
& \leq K_{T}\left[\left\|A^{-\beta}\right\| L_{g}+n M L_{I}+M n L_{g}\left(2+L_{I}\right)\right] \\
& \quad \times\left\|x_{1}-x_{2}\right\|_{T} .
\end{aligned}
$$

Hence, it implies that $Q_{1}$ satisfies the Lipschitz condition with Lipschitz constant $L$, where $L=K_{T}\left[\left\|A^{-\beta}\right\| L_{g}+n M L_{I}+\right.$ $\left.M n L_{g}\left(2+L_{I}\right)\right]$.

Therefore, from Lemma 9 (vii), we have that for any bounded set $B \in \mathscr{P} \mathscr{C}$

$$
\chi_{\mathscr{P} \mathscr{C}}\left(Q_{1} B\right) \leq L \chi_{\mathscr{P} \mathscr{C}}(B) .
$$

Next, we show that $Q_{2}$ is a $\chi$-contraction. Let $B$ be an arbitrary bounded subset $S(T)$. Since $S_{q}(t)$ is equicontinuous solution operator, therefore $S_{q}(t-s) f\left(s, u_{s}+z_{s}\right)$ is piecewise continuous. From Lemma 9, we have that for any bounded set $B \in \mathscr{P} \mathscr{C}$,

$$
\begin{aligned}
\chi\left(Q_{2} B(t)\right) & =\chi\left(\int_{0}^{t} S_{q}(t-s) f\left(s, B_{s}+z_{s}\right) d s\right) \\
& \leq \int_{0}^{t} \eta(s) \sup _{-\infty<\omega \leq 0} \chi(B(s+\omega)+z(s+\omega)) d s
\end{aligned}
$$

$$
\begin{aligned}
& \leq \int_{0}^{t} \eta(s) \sup _{0 \leq \tau \leq s} \chi(B(\tau)) d s \\
& \leq \chi_{\mathscr{P} \mathscr{C}}(B) \int_{0}^{t} \eta(s) d s, \quad t \in[0, T] .
\end{aligned}
$$

Thus for any bounded set $B \in \mathscr{P} \mathscr{C}$

$$
\begin{aligned}
\chi_{\mathscr{P C}}(Q B) & =\chi_{\mathscr{P C}}\left(Q_{1} B+Q_{2} B\right) \\
& \leq \chi_{\mathscr{P} \mathscr{C}}\left(Q_{1} B\right)+\chi_{\mathscr{P} \mathscr{C}}\left(Q_{2} B\right) \\
& \leq\left(L+\int_{0}^{t} \eta(s) d s\right) \chi_{\mathscr{P} \mathscr{C}}(B) .
\end{aligned}
$$

By the assumption (H2), we obtain that $\chi_{\mathscr{P} \mathscr{C}}(Q B)<\chi_{\mathscr{P} \mathscr{C}}(B)$; that is, $Q$ is a contraction. Therefore, $Q$ has at least one fixed point in $B$ by Darbo fixed point theorem. Let $u$ be a fixed point of $Q$ on $S(T)$, then $y=u+z$ is a mild solution for (1).

Theorem 18. Suppose that $(H f),(H g)$, and $(H I)$ are satisfied and

$$
\begin{aligned}
K_{T}\left[\left\|A^{-\beta}\right\| C_{1}+n M L_{1}\left(1+L_{g}\right)\right] \\
\quad+\int_{0}^{T} m_{f}(s) d s \lim _{\tau \rightarrow \infty} \sup \frac{\Omega(\tau)}{\tau}<1 .
\end{aligned}
$$

Then, there exists a mild solution for the impulsive problem (1).

Proof. Proceeding as in the proof of Theorem 17, we infer that $Q$ defined by (34) is continuous from $S(T)$ into $S(T)$. Next we indicate that there exists $r>0$ such that $Q\left(B_{r}\right) \subset B_{r}$, where $B$ is defined by $B_{r}=\left\{u \in S(T):\|u\|_{T} \leq r\right\}$. To this end, let us assume that assertion is false, then for any $r>0$ there exists $u_{r} \in B_{r}$ and $t_{r} \in J$ such that $r<\left\|Q u_{r}\left(t_{r}\right)\right\|$. Therefore, for $t_{r} \in\left[0, t_{1}\right]$ and $u_{r} \in B_{r}$,

$$
\begin{aligned}
r< & \left\|Q u_{r}\left(t_{r}\right)\right\| \leq M\|g(t, \varphi)\|+\left\|g\left(t, u_{r t_{r}}+z_{t_{r}}\right)\right\| \\
& +M \int_{0}^{t} m_{f}(s) \Omega\left(\left\|u_{r s}+z_{s}\right\|\right) d s, \\
\leq & M\|g(t, \varphi)\|+\left\|A^{-\beta}\right\|\left(C_{1}\left\|u_{r t_{r}}+z_{t_{r}}\right\|_{\mathscr{P}}+C_{2}\right) \\
& +M \int_{0}^{t} m_{f}(s) \Omega\left(\left\|u_{r_{S}}+z_{s}\right\|\right) d s, \\
\leq & M\|g(t, \varphi)\|+\left\|A^{-\beta}\right\| \\
& \times\left(C_{1}\left(K_{T} M H+N_{T}\right)\|\varphi\|_{\mathscr{P}}+C_{1} K_{T} r+C_{2}\right) \\
& +M \int_{0}^{t} m_{f}(s) d s \Omega\left(\left(K_{T} M H+N_{T}\right)\|\varphi\|_{\mathscr{P}}+K_{T} r\right) \\
= & r_{0} .
\end{aligned}
$$


For $t_{r} \in\left(t_{1}, t_{2}\right]$, we have

$$
\begin{aligned}
r< & \left\|Q u_{r}\left(t_{r}\right)\right\| \leq M\|g(t, \varphi)\| \\
& +\left\|A^{-\beta}\right\|\left(C_{1}\left\|u_{r t_{r}}+z_{t_{r}}\right\|_{\mathscr{P}}+C_{2}\right) \\
& +M\left(L_{1}\left\|u_{r t_{r}}+z_{t_{r}}\right\|_{\mathscr{P}}+L_{2}\right) \\
& +M L_{g}\left(L_{1}\left\|u_{r t_{r}}+z_{t_{r}}\right\|_{\mathscr{P}}+L_{2}\right) \\
& +M \int_{0}^{t} m_{f}(s) \Omega\left(\left\|u_{r_{s}}+z_{s}\right\|\right) d s, \\
\leq & M\|g(t, \varphi)\|+\left\|A^{-\beta}\right\|_{1} \\
& \times\left(C_{1}\left(K_{T} M H+N_{T}\right)\|\varphi\|_{\mathscr{P}}+C_{1} K_{T} r+C_{2}\right) \\
& +M\left(L_{1}\left(K_{T} M H+N_{T}\right)\|\varphi\|_{\mathscr{P}}+L_{1} K_{T} r+L_{2}\right) \\
& +M L_{g}\left(L_{1}\left(K_{T} M H+N_{T}\right)\|\varphi\|_{\mathscr{P}}+L_{1} K_{T} r+L_{2}\right) \\
& +M \int_{0}^{t} m_{f}(s) d s \Omega\left(\left(K_{T} M H+N_{T}\right)\|\varphi\|_{\mathscr{P}}+K_{T} r\right), \\
= & r_{1} \cdot
\end{aligned}
$$

For $t_{r} \in\left(t_{n}, T\right]$, we have

$$
\begin{aligned}
r< & \left\|Q u_{r}\left(t_{r}\right)\right\| \leq M\|g(t, \varphi)\| \\
& +\left\|A^{-\beta}\right\|\left(C_{1}\left(K_{T} M H+N_{T}\right)\|\varphi\|_{\mathscr{P}}+C_{1} K_{T} r+C_{2}\right) \\
& +n M\left(L_{1}\left(K_{T} M H+N_{T}\right)\|\varphi\|_{\mathscr{P}}+L_{1} K_{T} r+L_{2}\right) \\
& +n M L_{g}\left(L_{1}\left(K_{T} M H+N_{T}\right)\|\varphi\|_{\mathscr{P}}+L_{1} K_{T} r+L_{2}\right) \\
& +M \int_{0}^{t} m_{f}(s) d s \Omega\left(\left(K_{T} M H+N_{T}\right)\|\varphi\|_{\mathscr{P}}+K_{T} r\right), \\
= & r_{n},
\end{aligned}
$$

which implies that $r<\max \left\{r_{0}, r_{1}, \ldots, r_{n}\right\}$. Therefore, we conclude that

$$
\begin{aligned}
r< & M\|g(t, \varphi)\|+\left\|A^{-\beta}\right\| \\
& \times\left(C_{1}\left(K_{T} M H+N_{T}\right)\|\varphi\|_{\mathscr{P}}+C_{1} K_{T} r+C_{2}\right) \\
& +n M\left(L_{1}\left(K_{T} M H+N_{T}\right)\|\varphi\|_{\mathscr{P}}+L_{1} K_{T} r+L_{2}\right) \\
& +n M L_{g}\left(L_{1}\left(K_{T} M H+N_{T}\right)\|\varphi\|_{\mathscr{P}}+L_{1} K_{T} r+L_{2}\right) \\
& +M \int_{0}^{t} m_{f}(s) d s \Omega\left(\left(K_{T} M H+N_{T}\right)\|\varphi\|_{\mathscr{P}}+K_{T} r\right) .
\end{aligned}
$$

We divide both the sides of (58) by $r$ and letting $r \rightarrow \infty$, we obtain that

$$
\begin{aligned}
1< & K_{T}\left[\left\|A^{-\beta}\right\| C_{1}+n M L_{1}+n M L_{g} L_{1}\right] \\
& +M \int_{0}^{T} m_{f}(s) d s \\
& \times \lim _{r \rightarrow \infty} \sup \frac{\Omega\left(\left(K_{T} M H+N_{T}\right)\|\varphi\|_{\mathscr{P}}+K_{T} r\right)}{r}, \\
< & K_{T}\left[\left\|A^{-\beta}\right\| C_{1}+n M L_{1}\left(1+L_{g}\right)\right] \\
& +\int_{0}^{T} m_{f}(s) d s \lim _{\tau \rightarrow \infty} \sup \frac{\Omega(\tau)}{\tau} .
\end{aligned}
$$

This contradicts the inequality (54). Hence, there exists a positive constant $r>0$ such that $Q\left(B_{r}\right) \subset B_{r}$. Moreover, by uniform continuity of the map $t \mapsto S_{q}(t)$ on $(0, T]$, we have that set $Q\left(B_{r}\right)$ is equicontinuous. As the proof of Theorem 17, we infer that system (1) has a mild solution.

\section{Example}

In this section, we consider an example to illustrate the application of the theory. Here we take the space $C_{0} \times L^{2}(h, X)$ as the phase space $\mathscr{P}$ (see, [7]).

Now we study the following fractional differential equation with infinite delay:

$$
\begin{gathered}
\frac{d^{q}}{d t^{q}}\left[u(t, x)+\int_{-\infty}^{t} \int_{0}^{\pi} b(t-s, \mu, x) u(s, \mu) d \mu d s\right] \\
=\frac{\partial^{2}}{d x^{2}}\left[u(t, x)+\int_{-\infty}^{t} \int_{0}^{\pi} b(t-s, \mu, x) u(s, \mu) d \mu d s\right] \\
+\mathscr{J}_{t}^{1-q} \int_{-\infty}^{t} f(t, t-s, x, u(s, x)) d s, \\
u(t, 0)=u(t, \pi)=0, \quad 0 \leq t \leq T, x \in[0, \pi], \\
u(\tau, x)=\varphi(\tau, x), \quad 0 \geq \tau, x \in[0, \pi], \\
\Delta u\left(t_{k}, x\right)=\int_{-\infty}^{t_{k}} a_{k}\left(t_{k}-s\right) u(s, x) d s, \quad k=1,2, \ldots, n,
\end{gathered}
$$

where $\varphi \in C_{0} \times L^{2}(h ; X)$ and $0<t_{1}<\cdots<t_{n}<t_{n+1}=T$ are prefixed numbers. Let $X=L^{2}[0, \pi]$ and consider the operator $A: D(A) \subset X \rightarrow X$ as $A v=v^{\prime \prime}$ with the domain

$D(A)=\left\{v \in X: v, v^{\prime}\right.$ are absolutely continuous and $v^{\prime \prime} \in X$,

$$
v(0)=0=v(\pi)\} .
$$


Then,

$$
A=\sum_{n=1}^{\infty} n^{2}\left(v, v_{n}\right) v_{n}, \quad v \in D(A),
$$

Here $v_{n}(x)=\sqrt{(2 / \pi)} \sin (n x), n \in \mathbb{N}$ is the orthogonal set of eigenvectors of $A$. It is clear that $A$ is the infinitesimal generator of an analytic semigroup $\{S(t)\}_{t \geq 0}$ in $X$ which is given by

$$
S(t) v=\sum_{n=1}^{\infty} e^{-n^{2} t}\left(v, v_{n}\right) v_{n}, \quad \forall v \in X, t>0 .
$$

By Theorem 3.1 in [27], we get that $A$ is the infinitesimal generator of solution operator $\left\{S_{q}(t)\right\}_{t \geq 0}$ and there exists a positive constant $M$ such that $\left\|S_{q}(t)\right\|_{\mathscr{L}(X)} \leq M$ for $t \in[0, T]$. The functions $b, f, a_{k}$ satisfy the following conditions:

(1) the functions $b(s, \mu, x),(\partial b / \partial x)(s, \mu, x)$ are measurable such that $b(s, \mu, \pi)=b(s, \mu, 0)=0$ and

$$
\begin{aligned}
& L_{g} \\
& \qquad \max \left\{\left(\int_{0}^{\pi} \int_{-\infty}^{0} \int_{0}^{\pi} \frac{1}{h(s)}\left(\frac{\partial^{i} b(s, \mu, x)}{\partial x^{i}}\right)^{2} d \mu d s d x\right)^{1 / 2}:\right. \\
& \qquad i=0,1\}<\infty ;
\end{aligned}
$$

(2) $f: \mathbb{R}^{4} \rightarrow \mathbb{R}$ is continuous and there exist a $\eta$ : $C\left(\mathbb{R}^{2}, \mathbb{R}\right)$ such that

$$
\left\|f\left(t_{1}, t_{2}, x, u\right)\right\| \leq \eta\left(t_{1}, t_{2}\right)\|u\|, \quad\left(t_{1}, t_{2}, x, u\right) \in \mathbb{R}^{4}
$$

(3) the functions $a_{k}:[0, \infty) \rightarrow \mathbb{R}, k=1,2, \ldots, n$ are continuous and

$$
L_{I}=\left(\int_{-\infty}^{0} \frac{\left(a_{k}(s)\right)^{2}}{h(s)} d s\right)^{1 / 2}<\infty .
$$

The impulsive system (60) might be reformulated as the abstract impulsive Cauchy problem (1) where

$$
\begin{gathered}
g(t, \phi)(x)=\int_{-\infty}^{0} \int_{0}^{\pi} b(s, y, x) \phi(s, y) d y d s, \\
F(t, \phi)(x)=\int_{-\infty}^{0} f(t, s, x, \phi(s, x)) d s, \\
I_{k}(\phi)(x)=\int_{-\infty}^{0} a_{k}(s) \phi(s, x) d s, \quad k=1, \ldots, n .
\end{gathered}
$$

It may be verified that $g, F$ satisfy the assumptions ( $\mathrm{Hf}),(\mathrm{Hg})$, and $(\mathrm{HI})$; that is, $g(t, \cdot)$ and $I_{k}, k=1, \ldots, n$ are bounded linear operators and the range of $g(\cdot)$ is contained in $X_{1 / 2}$, $\left\|A^{1 / 2} g(t, \cdot)\right\| \leq L_{g},\left\|I_{k}\right\| \leq L_{I}, k=1, \ldots, n$, and $\|F(t, \phi)\| \leq$ $m_{F}(t)\|\phi\|_{\mathscr{P}}$ for every $t \in[0, T]$, where $m_{F}(t)$ is defined as $m_{F}(t)=\left(\int_{-\infty}^{0} \eta(t, s)^{2} / h(s) d s\right)^{1 / 2}$. Applying Theorem 17, we obtain that problem (60) has a mild solution.

\section{Conflict of Interests}

The authors declare that there is no conflict of interests regarding the publication of this paper.

\section{Acknowledgments}

The authors would like to thank the referee for valuable comments and suggestions. The work of the first author is supported by the UGC (University Grants Commission, India) under Grant no. (6405-11-061).nt.

\section{References}

[1] I. Podlubny, Fractional Differential Equations, Academic Press, New York, NY, USA, 1993.

[2] K. S. Miller and B. Ross, An Introduction to the Fractional Calculus and Fractional Differential Equations, John Wiley \& Sons, New York, NY, USA, 1993.

[3] S. G. Samko, A. A. Kilbas, and O. I. Marichev, Fractional Integrals and Derivatives: Theory and Applications, Gordon and Breach Science Publishers, Yverdon, Switzerland, 1993.

[4] A. A. Kilbas, H. M. Srivastava, and J. J. Trujillo, Theory and Applications of Fractional Differential Equations, Elsevier, Amsterdam, The Netherlands, 2006.

[5] J. K. Hale and J. Kato, "Phase space for retarded equations with infinite delay," Funkcialaj Ekvacioj, vol. 21, no. 1, pp. 11-41, 1978.

[6] Y. Hino, S. Murakami, and T. Naito, Functional-Differential Equations with Infinite Delay, vol. 1473 of Lecture Notes in Mathematics, Springer, Berlin, Germany, 1991.

[7] E. M. Hernández, M. Rabello, and H. R. Henríquez, "Existence of solutions for impulsive partial neutral functional differential equations," Journal of Mathematical Analysis and Applications, vol. 331, no. 2, pp. 1135-1158, 2007.

[8] E. Hernández and H. R. Henríquez, "Existence results for partial neutral functional-differential equations with unbounded delay," Journal of Mathematical Analysis and Applications, vol. 221, no. 2, pp. 452-475, 1998.

[9] F. Li, "An existence result for fractional differential equations of neutral type with infinite delay," Electronic Journal of Qualitative Theory of Differential Equations, vol. 52, pp. 1-15, 2011.

[10] F. Li and J. Zhang, "Existence of mild solutions to fractional integrodifferential equations of neutral type with infinite delay," Advances in Difference Equations, vol. 2011, Article ID 963463, 15 pages, 2011.

[11] R. P. Agarwal, M. Benchohra, and S. Hamani, "A survey on existence results for boundary value problems of nonlinear fractional differential equations and inclusions," Acta Applicandae Mathematicae, vol. 109, no. 3, pp. 973-1033, 2010.

[12] J. Dabas and A. Chauhan, "Existence and uniqueness of mild solution for an impulsive neutral fractional integro-differential equation with infinite delay," Mathematical and Computer Modelling, vol. 57, no. 3-4, pp. 754-763, 2013.

[13] J. Wang, M. Fečkan, and Y. Zhou, "On the new concept of solutions and existence results for impulsive fractional evolution equations," Dynamics of Partial Differential Equations, vol. 8, no. 4, pp. 345-361, 2011.

[14] M. Benchohra, J. Henderson, and S. Ntouyas, Impulsive Differential Equations and Inclusions, vol. 2 of Contemporary Mathematics and Its Applications, Hindawi, New York, NY, USA, 2006. 
[15] M. Fečkan, Y. Zhou, and J. Wang, "On the concept and existence of solution for impulsive fractional differential equations," Communications in Nonlinear Science and Numerical Simulation, vol. 17, no. 7, pp. 3050-3060, 2012.

[16] V. Lakshmikantham, D. D. Bainnov, and P. S. Simeonov, Theory of Impulsive Differential Equations, Series in Modern Applied Mathematics, World Scientific Publishing, Teaneck, NJ, USA, 1989.

[17] Y.-K. Chang, A. Anguraj, and M. M. Arjunan, "Existence results for impulsive neutral functional differential equations with infinite delay," Nonlinear Analysis: Hybrid Systems, vol. 2, no. 1, pp. 209-218, 2008.

[18] Y.-K. Chang, V. Kavitha, and M. M. Arjunan, "Existence results for impulsive neutral differential and integrodifferential equations with nonlocal conditions via fractional operators," Nonlinear Analysis: Hybrid Systems, vol. 4, no. 1, pp. 32-43, 2010.

[19] J. Banaś and K. Goebel, Measures of Noncompactness in Banach Spaces, Lecture Notes in Pure and Applied Mathematics, Marcel Dekker, New York, NY, USA, 1980.

[20] R. R. Akhmerov, M. I. Kamenskiŭ, A. S. Potapov, A. E. Rodkina, and B. N. Sadovski1̆, Measures of Noncompactness and Condensing Operators, Birkhäuser, Berlin, Germany, 1992.

[21] S. Ji and G. Li, "A unified approach to nonlocal impulsive differential equations with the measure of noncompactness," Advances in Difference Equations, vol. 2012, article 182, 14 pages, 2012.

[22] P. Chen and Y. Li, "Nonlocal problem for fractional evolution equations of mixed type with the measure of noncompactness," Abstract and Applied Analysis, vol. 2013, Article ID 784816, 12 pages, 2013.

[23] R. P. Agarwal, M. Benchohra, and D. Seba, "On the application of measure of noncompactness to the existence of solutions for fractional differential equations," Results in Mathematics, vol. 55, no. 3-4, pp. 221-230, 2009.

[24] X. Xue, "Nonlocal nonlinear differential equations with a measure of noncompactness in Banach spaces," Nonlinear Analysis: Theory, Methods and Applications, vol. 70, no. 7, pp. 2593-2601, 2009.

[25] Z. Fan and G. Mophou, "Nonlocal problems for fractional differential equations via resolvent operators," International Journal of Differential Equations, vol. 2013, Article ID 490673, 9 pages, 2013.

[26] A. Pazy, Semigroups of Linear Operators and Applications to Partial Differential Equations, Springer, New York, NY, USA, 1983.

[27] E. Bazhlekova, Fractional evolution equations in Banach spaces [Ph.D. thesis], Eindhoven University of Technology, 2001.

[28] R. P. Agarwal, M. Meehan, and D. O’Regan, Fixed Point Theory and Applications, Cambridge Tracts in Mathematics, Cambridge University Press, New York, NY, USA, 2001.

[29] R. Ye, "Existence of solutions for impulsive partial neutral functional differential equation with infinite delay," Nonlinear Analysis: Theory, Methods and Applications, vol. 73, no. 1, pp. 155-162, 2010. 


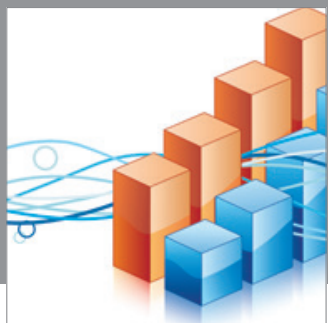

Advances in

Operations Research

mansans

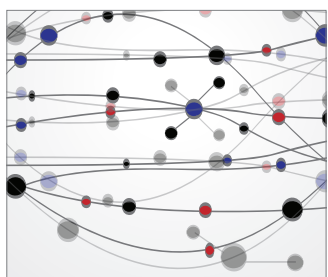

The Scientific World Journal
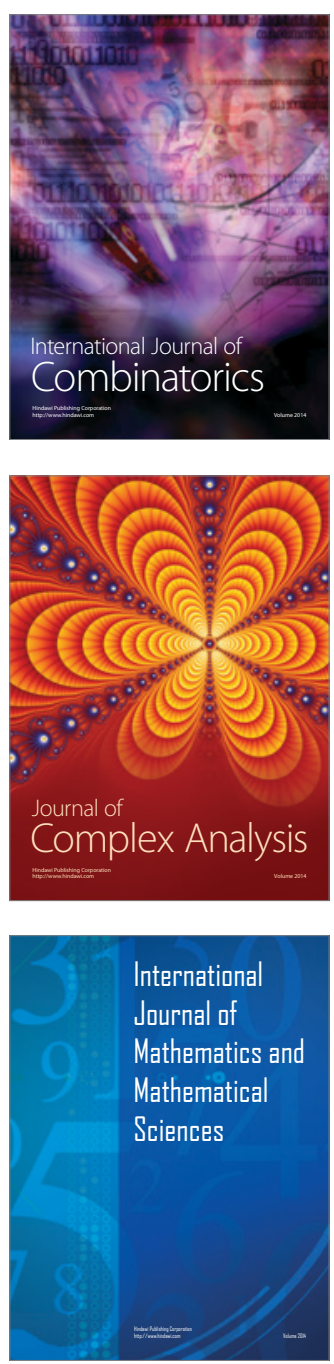
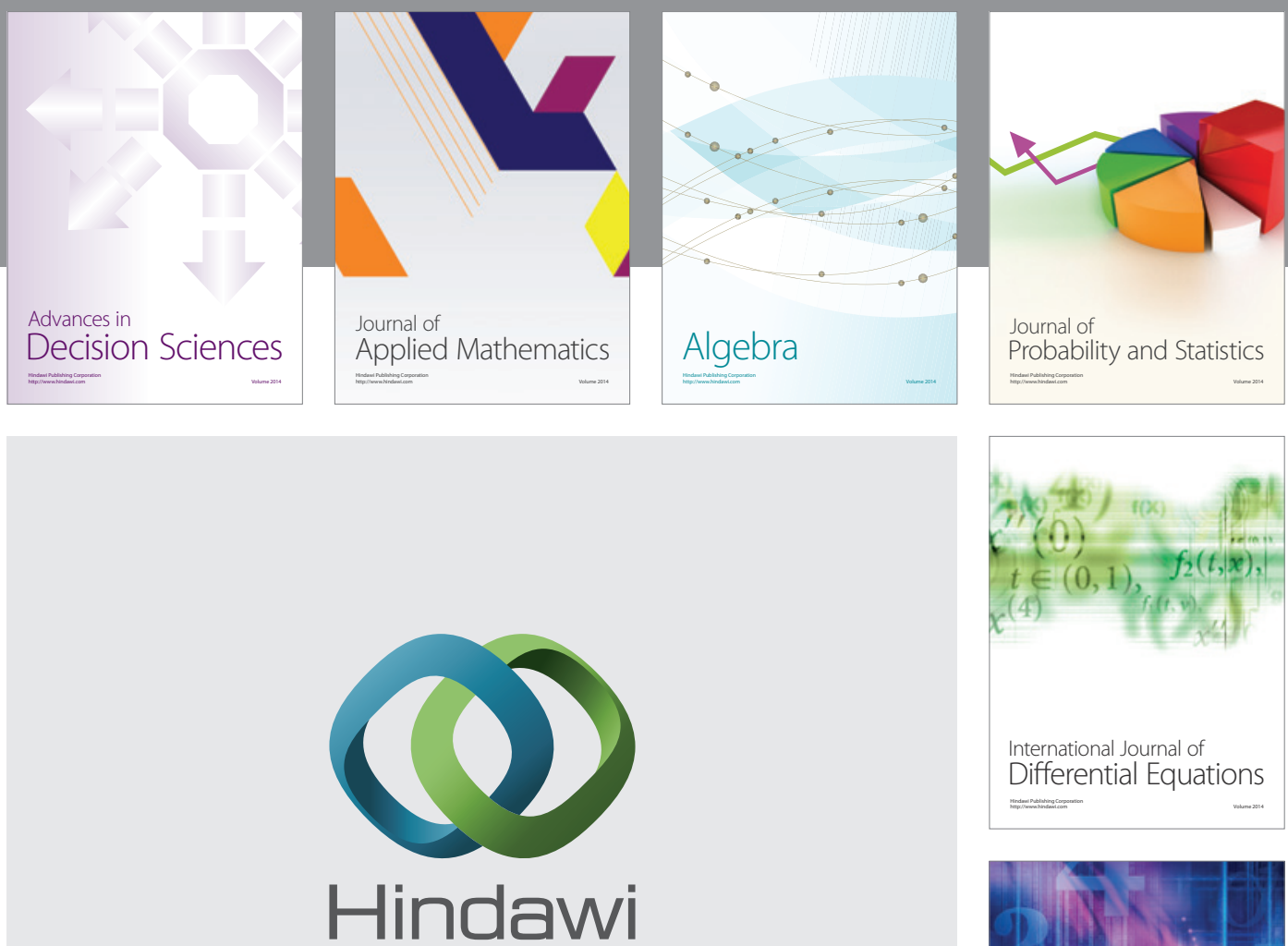

Submit your manuscripts at http://www.hindawi.com
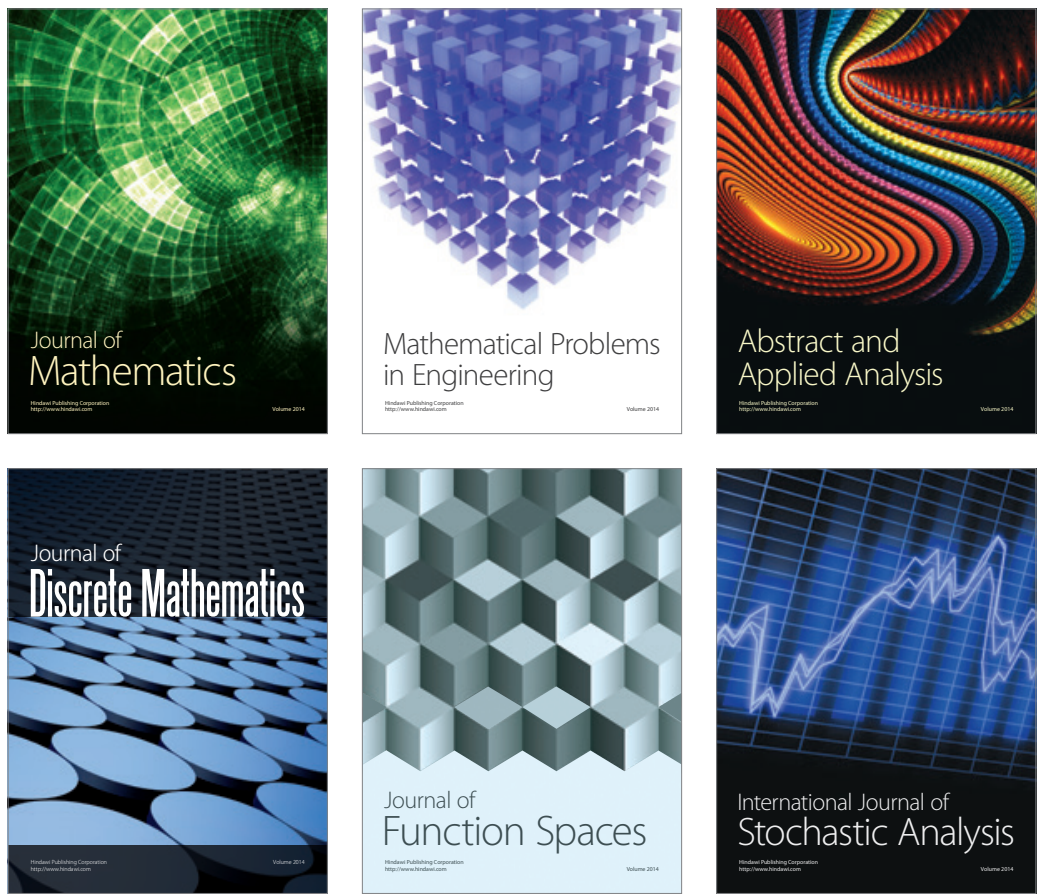

Journal of

Function Spaces

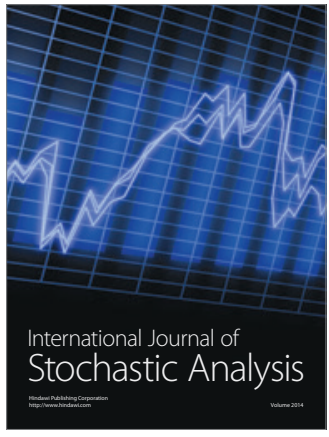

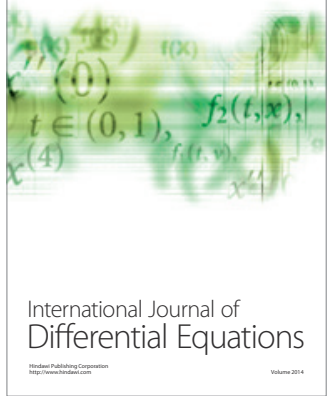
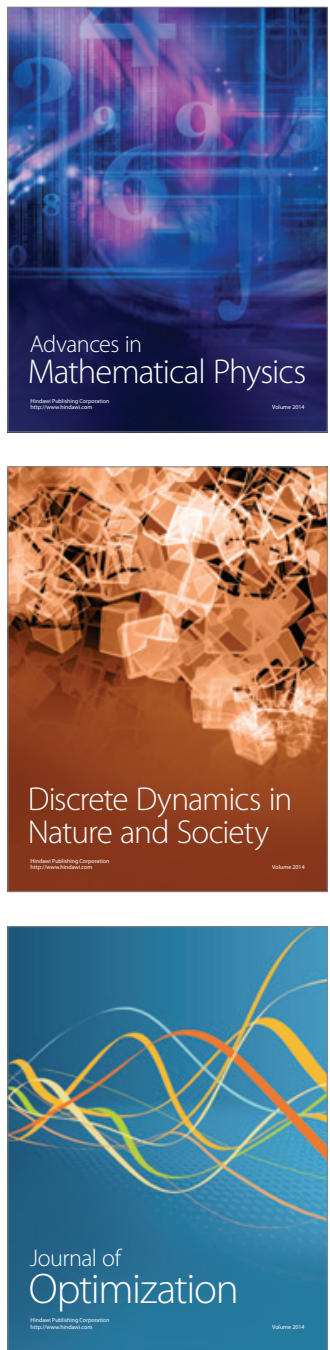